\title{
The in vitro effects of faropenem on lower respiratory tract pathogens isolated in the United Kingdom
}

\author{
F. Walsh*, A.K.B. Amyes, S.G.B. Amyes \\ Molecular Chemotherapy, Medical Microbiology, Medical School, University of Edinburgh, Teviot Place, Edinburgh EH8 9AG, UK
}

Received 30 July 2002; accepted 7 January 2003

\begin{abstract}
Faropenem is a new oral penem with a structure different from current $\beta$-lactams including carbapenems. The susceptibility of Streptococcus pneumoniae, Haemophilus influenzae and Moraxella catarrhalis to faropenem, a macrolide, a $\beta$-lactam, a $\beta$-lactam/ $\beta$ lactamase inhibitor combination and two fluoroquinolones was investigated. $S$. pneumoniae was the most susceptible of the three species to faropenem. The $\mathrm{MIC}_{90} \mathrm{~s}$ of faropenem against $M$. catarrhalis and $H$. influenzae were $0.5 \mathrm{and} 1 \mathrm{mg} / \mathrm{l}$, respectively. They were similar to amoxiclav ( $\mathrm{MIC}_{90} \mathrm{~s}$ of 0.25 and $0.5 \mathrm{mg} / \mathrm{l}$ ). The quinolones showed strong activity against $H$. influenzae. A cluster analysis of the activities of amoxycillin and faropenem demonstrated a direct relationship between the two antimicrobial agent's activities and resistance profiles against both $S$. pneumoniae and $H$. influenzae.
\end{abstract}

(C) 2003 Elsevier Science B.V. and the International Society of Chemotherapy. All rights reserved.

Keywords: Faropenem; Streptococcus pneumoniae; MIC

\section{Introduction}

Faropenem is a new oral penem antimicrobial agent, which has an unsaturated thiazole ring and is a structural hybrid between the penicillin and carbapenem nucleus (Fig. 1). It is characterised by potent penicillin binding protein activity and $\beta$-lactamase stability. The antibacterial spectrum of faropenem includes Grampositive, Gram-negative and some anaerobic bacteria [1]. In common with other $\beta$-lactams, target modifications via altered penicillin-binding proteins (PBPs) have previously been shown to increase the minimum inhibitory concentration (MIC) of faropenem for respiratory tract infections compared with wild type strains [2].

Faropenem typically shows high affinity for PBPs compared with other agents. Respiratory tract infections are a major cause of morbidity and mortality in the community and hospitals. Streptococcus pneumoniae, Moraxella catarrhalis and Haemophilus influenzae are common causes of lower respiratory tract infections

\footnotetext{
* Corresponding author. Tel.: +44-131-650-8270; fax: +44-131651-1385.

E-mail address: fwalsh@staffmail.ed.ac.uk (F. Walsh).
}

(LRTIs) and upper respiratory tract infections (URTIs). Worldwide, the annual incidence of community-acquired pneumonia (CAP) is estimated to be 1.1-4.0 per 1000 population. In the UK CAP accounts for 5$12 \%$ of cases of LRTIs among adults approximately 20 $42 \%$ of which require hospitalisation [3].

This study compared the in vitro activity of faropenem against $S$. pneumoniae, $M$. catarrhalis and $H$. influenzae isolated in the UK with amoxycillin, amoxiclav, clarithromycin, levofloxacin and ciprofloxacin activity.

\section{Materials and methods}

\subsection{Antimicrobial agents}

The following antimicrobial agents were studied: faropenem and ciprofloxacin (Bayer), amoxycillin (CP Pharmaceuticals), amoxiclav (SmithKline Beecham Pharmaceuticals and CP Pharmaceuticals), clarithromycin (Abbott) and levofloxacin (Aventis Pharma Ltd.). The antimicrobial agents were stored and prepared according to the suppliers' instructions. The amoxiclav 


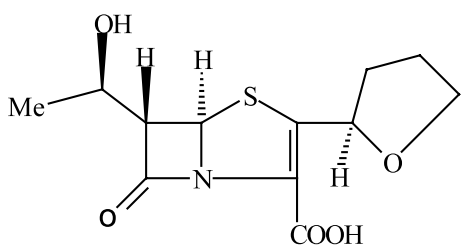

Fig. 1. Faropenem chemical structure.

consisted of fixed concentrations of $2 \mathrm{mg} / \mathrm{l}$ of clavulanic acid per plate and doubling dilutions of amoxycillin [4].

\subsection{Bacterial strains}

$S$. pneumoniae $(n=100)$ from Edinburgh and Leeds, M. catarrhalis (100) from Edinburgh, Leeds and Wales and $H$. influenzae (100) from Edinburgh and Glasgow, collected between 1997 and 2000, were used in this study. The control strains comprised $S$. pneumoniae NCTC 13593, Staphylococcus aureus NCTC 6571, H. influenzae NCTC 11931 and a laboratory reference strain of $M$. catarrhalis.

\subsection{Susceptibility testing}

MICs were determined by standard agar dilution methods according to the BSAC guidelines for sensitivity testing [4] on Columbia agar (Oxoid, Basingstoke) supplemented with 5\% defibrinated horse blood for $S$. pneumoniae and $M$. catarrhalis and chocolate Columbia agar plates for $H$. influenzae.

A final concentration of $10^{5} \mathrm{cfu}$ per spot of $S$. pneumoniae and $10^{4} \mathrm{cfu}$ per spot of $H$. influenzae and M. catarrhalis were inoculated onto each plate. The plates were incubated aerobically.

\section{Results}

The MICs of faropenem and the comparators are given in Table 1.

For $S$. pneumoniae, faropenem had the lowest $\mathrm{MIC}_{50}$ $(0.008 \mathrm{mg} / \mathrm{l})$ and $\mathrm{MIC}_{90}(0.25 \mathrm{mg} / \mathrm{l})$ of all the antimicrobial agents tested. The faropenem modal MIC against $S$. pneumoniae was $0.008 \mathrm{mg} / 1$ for 39 isolates, which was also the lowest concentration compared with $0.016 \mathrm{mg} / 1$ (37 isolates) for amoxiclav, $0.016-0.032 \mathrm{mg} / \mathrm{l}$ for amoxycillin against 70 isolates, $0.032 \mathrm{mg} / \mathrm{l}$ (42 isolates) for clarithromycin and $1 \mathrm{mg} / \mathrm{l}$ for both levofloxacin (48 isolates) and ciprofloxacin (28 isolates).

Faropenem had the second largest $\mathrm{MIC}_{90}$ and the second highest range endpoint against $M$. catarrhalis . The quinolones levofloxacin and ciprofloxacin had the lowest $\mathrm{MIC}_{90} \mathrm{~s}$ at $0.06 \mathrm{mg} / \mathrm{l}$. The modal MICs of faropenem were $0.06 \mathrm{mg} / \mathrm{l}$, for 27 isolates and $0.25 \mathrm{mg} /$ 1 for 25 isolates of $M$. catarrhalis. Levofloxacin and ciprofloxacin inhibited 66 and 73 strains of M. catarrhalis, respectively, at $0.032 \mathrm{mg} / \mathrm{l}$. The mode MIC of clarithromycin was $0.12 \mathrm{mg} / \mathrm{l}$, which inhibited 54 isolates. Amoxiclav did not have a mode concentration against $M$. catarrhalis. Amoxiclav inhibited 17 isolates at $0.004,0.008$ and $0.6 \mathrm{mg} / \mathrm{l} ; 16$ isolates had amoxiclav MICs of $0.12 \mathrm{mg} / 1,12$ isolates had MICs of $0.002 \mathrm{mg} / 1$, nine had MICs of $0.25 \mathrm{mg} / \mathrm{l}$, eight were inhibited by $0.032 \mathrm{mg} / \mathrm{l}$ and four isolates were inhibited by $0.016 \mathrm{mg} /$ 1. The mode concentration of amoxycillin was between 1 and $4 \mathrm{mg} / \mathrm{l}$ and inhibited 61 isolates.

The $\mathrm{MIC}_{50}$ and $\mathrm{MIC}_{90}$ of faropenem for $H$. influenzae were 0.5 and $1 \mathrm{mg} / \mathrm{l}$, respectively. Clarithromycin had an $\mathrm{MIC}_{50}$ and $\mathrm{MIC}_{90}$ of 4 and $8 \mathrm{mg} / \mathrm{l}$. Both quinolones had $\mathrm{MIC}_{50}$ and $\mathrm{MIC}_{90} \mathrm{~S}$ of six doubling dilutions lower than faropenem. However, the results of faropenem were

Table 1

MIC values of the antimicrobial agents

\begin{tabular}{llllll}
\hline Organism (number) & Antimicrobial agent & $\mathrm{MIC}_{50}(\mathrm{mg} / \mathrm{l})$ & $\mathrm{MIC}_{90}(\mathrm{mg} / \mathrm{l})$ & Range $(\mathrm{mg} / \mathrm{l})$ & Mode MIC (mg/l), [number of strains] \\
\hline S. pneumoniae (100) & Faropenem & 0.008 & 0.25 & $0.002-1$ & $0.008[39]$ \\
& Amoxycillin & 0.032 & 0.5 & $0.004-2$ & $0.016[36] / 0.032[34]$ \\
& Amoxiclav & 0.016 & 0.5 & $0.004-2$ & $0.016[37]$ \\
& Clarithromycin & 0.06 & 2 & $0.008->32$ & $0.032[42]$ \\
& Levofloxacin & 1 & 2 & $0.12-2$ & $1[48]$ \\
& Ciprofloxacin & 1 & 4 & $0.032-8$ & $1[28]$ \\
M. catarrhalis (100) & Faropenem & 0.12 & 0.5 & $0.032-1$ & $0.06[27] / 0.25[25]$ \\
& Amoxycillin & 1 & 4 & $0.016-8$ & $1[16] / 2[20] / 4[25]$ \\
& Amoxiclav & 0.016 & 0.25 & $0.002-0.25$ & $0.004[17] / 0.008[17], 0.06[17] / 0.12[16]$ \\
& Clarithromycin & 0.12 & 0.12 & $0.032-0.25$ & $0.12[54]$ \\
& Levofloxacin & 0.032 & 0.06 & $0.016-0.12$ & $0.032[66]$ \\
& Ciprofloxacin & 0.032 & 0.06 & $0.016-0.06$ & $0.032[73]$ \\
& Faropenem & 0.5 & 1 & $0.06-4$ & $0.5[48]$ \\
& Amoxycillin & 0.5 & 4 & $<0.032-32$ & $0.5[50]$ \\
& Amoxiclav & 0.25 & 0.5 & $0.016-2$ & $0.25[33] / 0.5[40]$ \\
& Clarithromycin & 4 & 8 & $<0.06-16$ & $8[36]$ \\
& Levofloxacin & 0.008 & 0.016 & $0.004-1$ & $0.008[43]$ \\
& Ciprofloxacin & 0.008 & 0.016 & $<0.004-2$ & $0.008[66]$ \\
\hline
\end{tabular}




\section{$\underline{\text { Streptococcus pneumoniae }}$}

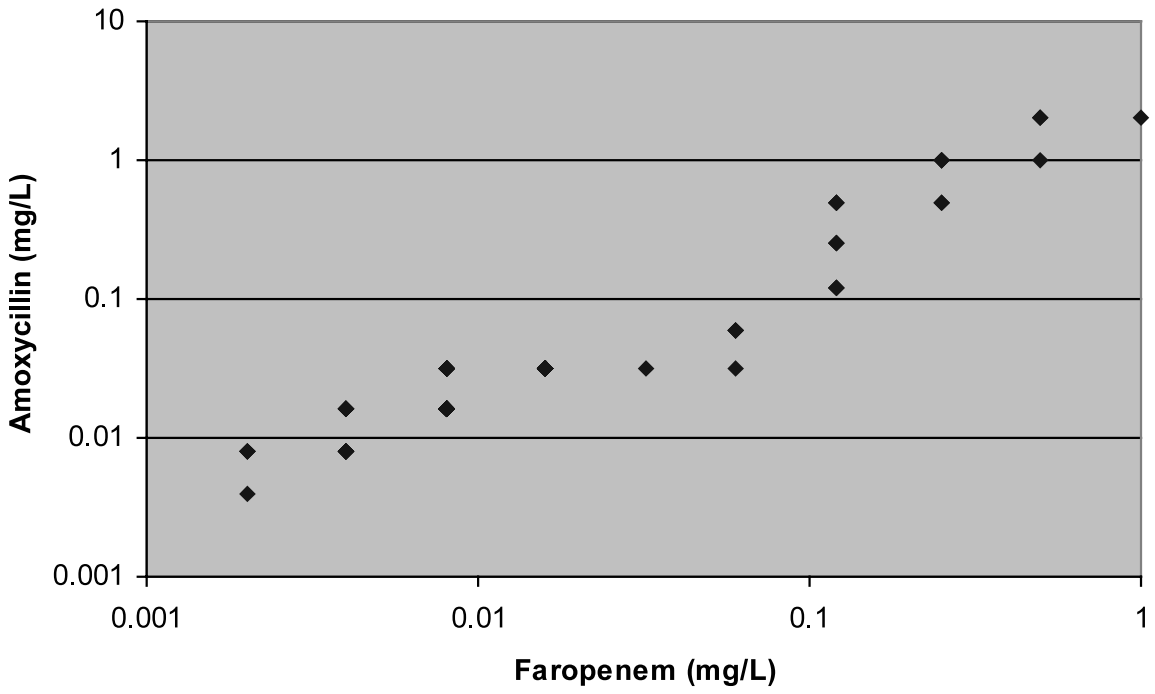

Moraxella catarrhalis

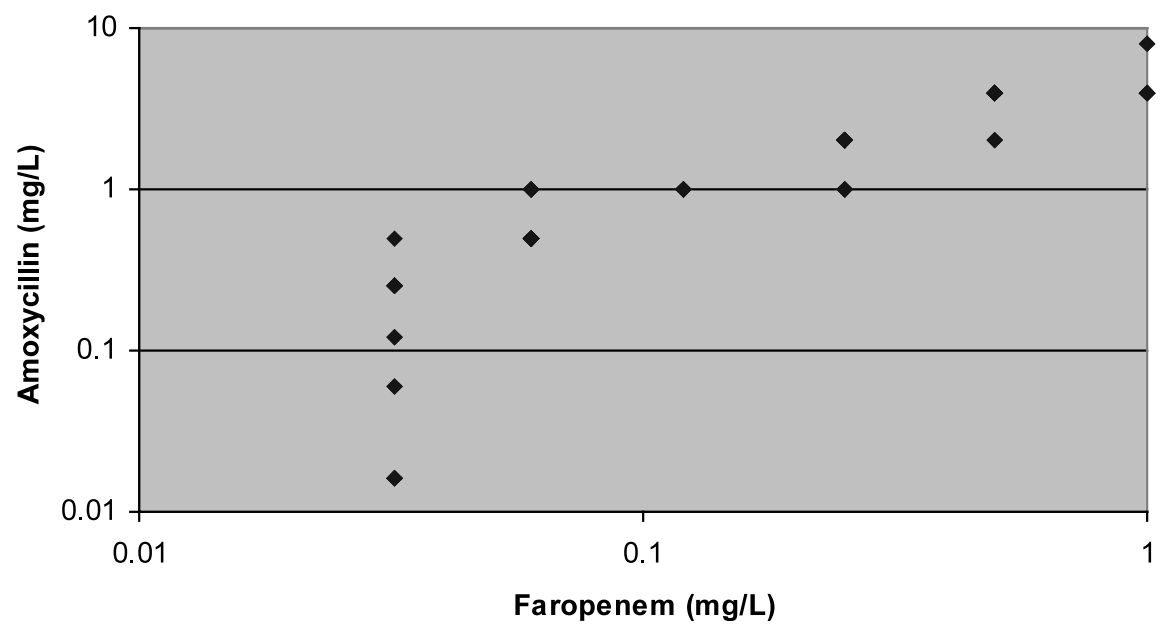

Haemophilus influenzae

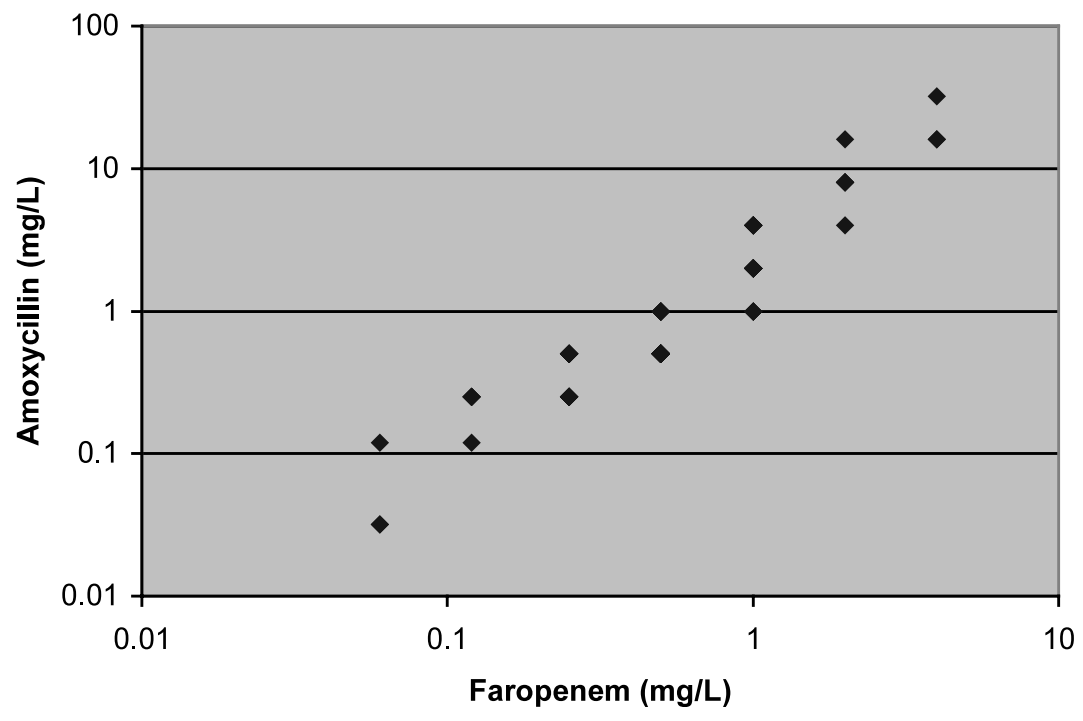

Fig. 2. Cluster analysis of amoxycillin and faropenem activity. 
comparable with amoxiclav, which had lower $\mathrm{MIC}_{50}$ and $\mathrm{MIC}_{90}$ values than faropenem, but only by one doubling dilution. The $\mathrm{MIC}_{50}$ and $\mathrm{MIC}_{90}$ of amoxycillin against $H$. influenzae were 0.5 and $4 \mathrm{mg} / \mathrm{l}$, respectively. The mode MIC of faropenem for $H$. influenzae was 0.5 $\mathrm{mg} / \mathrm{l}$ for 48 isolates compared with $0.008 \mathrm{mg} / 1$ for levofloxacin and ciprofloxacin against 43 and 66 isolates, respectively.

The results suggest that there might be a direct correlation between the sensitivity to amoxycillin and to faropenem. So a cluster analysis of faropenem and amoxycillin MICs against $S$. pneumoniae, $M$. catarrhalis and $H$. influenzae was performed (Fig. 2). The two graphs have the same distribution patterns and both indicate that there is a direct relationship between amoxycillin and faropenem inhibition of $S$. pneumoniae and $H$. influenzae. As resistance to amoxycillin increases, there is a proportional increase in resistance to faropenem. The correlation with $M$. catarrhalis is less pronounced and does not occur until high levels of penicillin resistance are reached. Thus, strains of $S$. pneumoniae and in many cases, $H$. influenzae isolates, resistant to amoxycillin due to alterations in penicillin binding proteins are also able to confer resistance to faropenem [7]. Those strains where $\beta$-lactamase resistance is more prominent, particularly $M$. catarrhalis show less resistance to faropenem.

\section{Discussion}

The faropenem results obtained in this study agree with previously reported data and indicate that it has significant activity $\left(\mathrm{MIC}_{90} \leq 1 \mathrm{mg} / \mathrm{l}\right)$ against respiratory tract infections such as $S$. pneumoniae, $M$. catarrhalis and $H$. influenzae [5,6]. The activity of faropenem was usually of a similar level to that of amoxiclav. This is perhaps not surprising as the clavulanic acid component of amoxiclav removes the $\beta$-lactamase contribution to amoxycillin resistance and faropenem is $\beta$-lactamase stable. This is emphasised by the correlation between amoxycillin and faropenem inhibition shown in Fig. 2. These cluster analyses show a direct relationship between faropenem and amoxycillin sensitivities demonstrating that faropenem was not capable of over-coming the resistance mechanisms arising from alteration of penicillin binding proteins in $S$. pneumoniae. The correlation was also quite clear in $H$. influenzae, where much of the amoxycillin resistance is mediated by mechanisms other than $\beta$-lactamase hydrolysis [7].
There is less obvious correlation in $M$. catarrhalis, where $\beta$-lactamase hydrolysis is a more prominent mechanism of amoxycillin hydrolysis [8]. In this case, there is a major increase in amoxycillin resistance without a significant change in faropenem resistance. It is only after a threshold is reached that further increases in amoxycillin resistance correspond with concomitant increases in faropenem resistance. As anticipated the two quinolones tested had lower $\mathrm{MIC}_{50}$ and MIC 90 values than all the agents tested for $H$. influenzae and all the agents except amoxiclav for $M$. catarrhalis. In these in vitro tests clarithromycin does not perform as well as faropenem against $S$. pneumoniae or $H$. influenzae. The results of faropenem demonstrate that it is a potent drug but these results suggest that the acquisition of penicillin resistance would have an adverse effect on faropenem in $S$. pneumoniae and $H$. influenzae but less so in $M$. catarhalis.

\section{Acknowledgements}

Faropenem was provided by Bayer A.G.

\section{References}

[1] Woodcock JM, Andrews JM, Brenwald NP, Ashby JP, Wise R. The in-vitro activity of faropenem, a novel oral penem. J Antimicrob Chemother 1997;39:35-43.

[2] Marchese A, Debbia EA, Bryskier A, Schito GC. Antimicrobial activity of faropenem, a new oral penem, against lower respiratory tract pathogens. Clin Microbiol Infect 1999;5:282-7.

[3] Finch R. Community-acquired pneumonia: the evolving challenge. Clin Microbiol Infect 2001;3(7 Suppl.):30-8.

[4] The British Society for Antimicrobial Chemotherapy. A guide to sensitivity testing, Journal of Antimicrobial Chemotherapy 1991;27:Suppl. D;1-30

[5] The British Society for Antimicrobial Chemotherapy. A guide to sensitivity testing, Journal of Antimicrobial Chemotherapy 1991;27:Suppl. D;1-30.

[6] Cormican MG, Jones RN. Evaluation of the in vitro activity of furopenem (SY5555 or SUN5555) against respiratory tract pathogens and $\beta$-lactamase producing bacteria. J Antimicrob Chemother 1995;35:535-9.

[7] Reid AJ, Simpson IN, Harper PB, Amyes SGB. Ampicillin resistance in $H$. influenzae: identification of resistance mechanisms. J Chemother 1987;20:645-56.

[8] Fung CP, Yeo SF, Livermore DM. Susceptibility of $M$. catarrhalis isolates to $\beta$-lactam antibiotics in relation to $\beta$-lactamase pattern. $\mathbf{J}$ Antimicrob Chemother 1994;33:215-22. 